\title{
Activation of chlorosilanes at ruthenium: a route to dihydrogen silyl complexes
}

\author{
Sébastien Lachaize, Ana Caballero, Laure Vendier and Sylviane Sabo-Etienne* \\ Laboratoire de Chimie de Coordination du CNRS, 205 route de Narbonne, 31077 Toulouse Cedex 04, France.
}

\section{Supplementary Information}

The optimized geometries (DFT/B3PW91) of the studied complexes and organic compounds are listed in this file (in a xyz file format) in the following order:

$\mathrm{S3Me}_{2} \mathrm{Cl}(\mathrm{R} 1=\mathrm{Me})$

$\mathrm{S}_{3 \mathrm{Me}} \mathrm{Cl}(\mathrm{R} 1=\mathrm{Cl})$

$\mathbf{S 3 M e C l}_{2}(\mathrm{R} 1=\mathrm{Me})$

$\mathrm{S}_{3 \mathrm{MeCl}}(\mathrm{R} 1=\mathrm{Cl})$

$\mathrm{S3Cl}_{3}$

S2

$\mathrm{TS3Me}_{2} \mathbf{C l}$ (rotation of the silyl group by ca $60^{\circ}$ )

$\mathbf{T S 3 M e C l}_{2}$ (rotation of the silyl group by ca $60^{\circ}$ )

$\mathrm{H}_{2}$

$\mathrm{HSiMe}_{2} \mathrm{Cl}$

$\mathrm{HSiMeCl}_{2}$

$\mathrm{HSiCl}_{3}$

The table with the main distances and angles of the DFT/B3LYP optimized geometries is given at the end (Table A) for comparison with the data reported in the article (see Table 2: DFT/B3PW91 level). Although the results are similar at the two calculation levels, the silicon and $\mathrm{H} 1$ positions given at the B3PW91 level are in better agreement with the X-ray and NMR data. Moreover, the potential energy surface for $\mathbf{S 3 C l}_{\mathbf{3}}$ is flatter with B3LYP, leading to the optimization of a second local minimum ( $\sigma$-silane isomer) at $+0.8 \mathrm{~kJ}^{\mathrm{mol}}{ }^{-1}$ above the $\sigma$ - 
dihydrogen isomer. The activation energy for isomerisation from $\sigma$-silane to $\sigma$-dihydrogen is roughly $+0.3 \mathrm{~kJ} \mathrm{~mol}^{-1}$. Such a small energy difference is not significant and thus prevent any further discussion. We thus preferred to use the B3PW91 data which are in better agreement with the experiments. 
$\mathrm{S} 3 \mathrm{Me}_{2} \mathrm{Cl}(\mathrm{R} 1=\mathrm{Me})$

Energy $=-202.6853993$ Hartree/particule

40

$\begin{array}{lrrr}\mathrm{Ru} & 0.00364 & 0.01087 & -0.00222 \\ \mathrm{Si} & -0.00234 & -0.01436 & 2.31531 \\ \mathrm{P} & 2.33697 & 0.00262 & -0.23969 \\ \mathrm{P} & -2.32575 & 0.08823 & -0.34328 \\ \mathrm{Cl} & -0.00749 & -2.22439 & -0.90114 \\ \mathrm{Cl} & -1.75074 & 0.80858 & 3.25105 \\ \mathrm{C} & 1.35422 & 0.84646 & 3.31206 \\ \mathrm{C} & -0.05424 & -1.83376 & 2.83638 \\ \mathrm{C} & 3.30530 & 1.50932 & 0.21971 \\ \mathrm{C} & 3.32356 & -1.37559 & 0.49404 \\ \mathrm{C} & 2.74156 & -0.16144 & -2.03733 \\ \mathrm{C} & -2.67801 & -0.14215 & -2.14428 \\ \mathrm{C} & -3.38313 & -1.20798 & 0.43171 \\ \mathrm{C} & -3.21894 & 1.65958 & 0.02575 \\ \mathrm{H} & 0.05972 & 1.49564 & -0.52475 \\ \mathrm{H} & -0.03398 & 1.36294 & 0.85290 \\ \mathrm{H} & 1.14574 & 0.71938 & 4.39144 \\ \mathrm{H} & 2.35191 & 0.41212 & 3.11143 \\ \mathrm{H} & 1.39603 & 1.93143 & 3.10357 \\ \mathrm{H} & -0.11647 & -1.90106 & 3.94061 \\ \mathrm{H} & 0.85418 & -2.36321 & 2.49494 \\ \mathrm{H} & -0.92360 & -2.35486 & 2.40076 \\ \mathrm{H} & 4.37038 & 1.40146 & -0.05675 \\ \mathrm{H} & 2.87913 & 2.37884 & -0.31080 \\ \mathrm{H} & 3.22955 & 1.69587 & 1.30346 \\ \mathrm{H} & 2.86054 & -2.32760 & 0.18216 \\ \mathrm{H} & 4.37581 & -1.34326 & 0.15620 \\ \mathrm{H} & 3.29471 & -1.31532 & 1.59559 \\ \mathrm{H} & 2.30145 & -1.10253 & -2.40871 \\ \mathrm{H} & 2.29011 & 0.68000 & -2.59212 \\ \mathrm{H} & 3.83464 & -0.16512 & -2.20533 \\ \mathrm{H} & -2.27178 & -1.11837 & -2.45906 \\ \mathrm{H} & -3.76467 & -0.10397 & -2.34580 \\ \mathrm{H} & -2.17279 & 0.65152 & -2.72271 \\ \mathrm{H} & -2.96597 & -2.19581 & 0.17092 \\ \mathrm{H} & -3.36156 & -1.08465 & 1.52786 \\ \mathrm{H} & -4.42705 & -1.13863 & 0.07391 \\ & -3.14405 & 1.87749 & 1.10382 \\ -2.73912 & 2.48110 & -0.53471 \\ \mathrm{H} & -4.28337 & 1.59184 & -0.26537\end{array}$


S3Me ${ }_{2} \mathrm{Cl}(\mathrm{R} 1=\mathrm{Cl})$

Energy $=-202.6828619$ Hartree/particule

40

$\begin{array}{lrrr}\mathrm{Ru} & -0.00037 & -0.00859 & 0.00609 \\ \mathrm{Si} & 0.00083 & 0.01516 & 2.32638 \\ \mathrm{P} & 2.33408 & -0.00221 & -0.30760 \\ \mathrm{P} & -2.33066 & 0.13818 & -0.30016 \\ \mathrm{Cl} & -0.05374 & -1.74407 & -1.66792 \\ \mathrm{Cl} & -0.07645 & -2.12691 & 2.40938 \\ \mathrm{C} & 1.51268 & 0.47098 & 3.36219 \\ \mathrm{C} & -1.47098 & 0.58016 & 3.36675 \\ \mathrm{C} & -3.25403 & 1.58476 & 0.39460 \\ \mathrm{C} & -3.34840 & -1.31509 & 0.20986 \\ \mathrm{C} & -2.76226 & 0.30591 & -2.08919 \\ \mathrm{C} & 3.35220 & 1.37286 & 0.40022 \\ \mathrm{C} & 3.26564 & -1.52268 & 0.17129 \\ \mathrm{C} & 2.76031 & 0.16688 & -2.09793 \\ \mathrm{H} & 0.04147 & 1.38199 & -0.69502 \\ \mathrm{H} & 0.04039 & 1.30445 & 0.96210 \\ \mathrm{H} & 1.34178 & 0.15946 & 4.41021 \\ \mathrm{H} & 2.43515 & -0.02691 & 3.01744 \\ \mathrm{H} & 1.68134 & 1.56572 & 3.35678 \\ \mathrm{H} & -1.31823 & 0.25971 & 4.41492 \\ \mathrm{H} & -2.42922 & 0.14991 & 3.02831 \\ \mathrm{H} & -1.56003 & 1.68425 & 3.35944 \\ \mathrm{H} & -3.22538 & 1.57999 & 1.49537 \\ \mathrm{H} & -2.77143 & 2.51221 & 0.03890 \\ \mathrm{H} & -4.30830 & 1.57592 & 0.06151 \\ \mathrm{H} & -4.41261 & -1.18328 & -0.05991 \\ \mathrm{H} & -2.94095 & -2.20424 & -0.30151 \\ \mathrm{H} & -3.25813 & -1.47621 & 1.29767 \\ \mathrm{H} & -2.35268 & -0.55850 & -2.63685 \\ \mathrm{H} & -3.85822 & 0.35929 & -2.22588 \\ \mathrm{H} & -2.29745 & 1.22398 & -2.48934 \\ \mathrm{H} & 4.39751 & 1.30982 & 0.04557 \\ \mathrm{H} & 2.91846 & 2.33470 & 0.07428 \\ \mathrm{H} & 3.34497 & 1.34434 & 1.50108 \\ \mathrm{H} & 2.79903 & -2.37815 & -0.34693 \\ \mathrm{H} & 4.33298 & -1.45213 & -0.10924 \\ \mathrm{H} & 3.17839 & -1.69287 & 1.25804 \\ \mathrm{H} & 2.28944 & -0.65934 & -2.65527 \\ \mathrm{H} & 2.35322 & 1.12034 & -2.47771 \\ & 3.85613 & 0.15038 & -2.24473\end{array}$


S3MeCl $2(\mathrm{R} 1=\mathrm{Me})$

Energy $=-210.2121816$ Hartree/particule

$\begin{array}{cccc}37 & & & \\ & & & \\ \mathrm{Ru} & 0.00103 & -0.01595 & 0.00238 \\ \mathrm{Si} & 0.00002 & 0.01008 & 2.28829 \\ \mathrm{P} & 2.34706 & -0.00209 & -0.25325 \\ \mathrm{P} & -2.34125 & -0.08709 & -0.27064 \\ \mathrm{Cl} & 0.04649 & -2.34784 & -0.52715 \\ \mathrm{Cl} & -1.66582 & 0.95475 & 3.21853 \\ \mathrm{C} & 0.05013 & -1.69643 & 3.07887 \\ \mathrm{Cl} & 1.60568 & 1.05327 & 3.21877 \\ \mathrm{C} & -3.31116 & 1.46428 & -0.03220 \\ \mathrm{C} & -3.30781 & -1.35309 & 0.65626 \\ \mathrm{C} & -2.71721 & -0.51232 & -2.03195 \\ \mathrm{C} & 2.74994 & -0.42236 & -2.00959 \\ \mathrm{C} & 3.35100 & -1.22844 & 0.68696 \\ \mathrm{C} & 3.25866 & 1.58434 & -0.01574 \\ \mathrm{H} & -0.01687 & 1.54270 & -0.42813 \\ \mathrm{H} & -0.04274 & 1.47493 & 0.64243 \\ \mathrm{H} & 0.04749 & -1.60342 & 4.18074 \\ \mathrm{H} & -0.82555 & -2.28787 & 2.75872 \\ \mathrm{H} & 0.95818 & -2.23702 & 2.75886 \\ \mathrm{H} & -3.20309 & 1.80708 & 1.01006 \\ \mathrm{H} & -2.91179 & 2.24443 & -0.70405 \\ \mathrm{H} & -4.38128 & 1.30375 & -0.25782 \\ \mathrm{H} & -3.31283 & -1.09396 & 1.72870 \\ \mathrm{H} & -4.34876 & -1.40739 & 0.28816 \\ \mathrm{H} & -2.81300 & -2.33018 & 0.52247 \\ \mathrm{H} & -3.80807 & -0.53171 & -2.21328 \\ \mathrm{H} & -2.25381 & 0.23481 & -2.70079 \\ \mathrm{H} & -2.28481 & -1.50215 & -2.25666 \\ \mathrm{H} & 2.34838 & -1.42542 & -2.23277 \\ \mathrm{H} & 2.26964 & 0.30796 & -2.68503 \\ \mathrm{H} & 3.84225 & -0.40991 & -2.18245 \\ \mathrm{H} & 3.33823 & -0.96466 & 1.75818 \\ \mathrm{H} & 2.89212 & -2.22297 & 0.55382 \\ \mathrm{H} & 4.39616 & -1.24769 & 0.32750 \\ \mathrm{H} & 3.13470 & 1.92553 & 1.02528 \\ \mathrm{H} & 4.33459 & 1.46269 & -0.23815 \\ \mathrm{H} & 2.83274 & 2.34762 & -0.69066\end{array}$


$\mathrm{S} 3 \mathrm{MeCl}_{2}(\mathrm{R} 1=\mathrm{Cl})$

Energy $=-210.208978$ Hartree/particule

37

$\begin{array}{cccc}\mathrm{Ru} & -0.00328 & -0.00115 & 0.00157 \\ \mathrm{Si} & 0.00407 & 0.00989 & 2.29289 \\ \mathrm{P} & 2.34406 & -0.00105 & -0.31212 \\ \mathrm{P} & -2.34599 & 0.11559 & -0.25002 \\ \mathrm{Cl} & -0.09372 & -1.81528 & -1.56426 \\ \mathrm{Cl} & 1.73108 & 0.66225 & 3.33420 \\ \mathrm{C} & -1.36068 & 0.63859 & 3.42182 \\ \mathrm{Cl} & -0.00028 & -2.09520 & 2.57276 \\ \mathrm{H} & 0.01882 & 1.42391 & -0.62894 \\ \mathrm{H} & 0.05707 & 1.32661 & 0.92816 \\ \mathrm{H} & -1.11519 & 0.36643 & 4.46442 \\ \mathrm{H} & -1.44524 & 1.73998 & 3.36391 \\ \mathrm{H} & -2.33857 & 0.18772 & 3.17529 \\ \mathrm{C} & 3.29890 & 1.48905 & 0.21384 \\ \mathrm{C} & 3.28324 & -1.41348 & 0.40826 \\ \mathrm{C} & 2.78903 & -0.08682 & -2.10267 \\ \mathrm{C} & -3.26872 & 1.55176 & 0.46244 \\ \mathrm{C} & -3.32696 & -1.34979 & 0.29209 \\ \mathrm{C} & -2.80681 & 0.26637 & -2.03268 \\ \mathrm{H} & 4.34517 & -1.38657 & 0.10155 \\ \mathrm{H} & 3.21533 & -1.37697 & 1.50862 \\ \mathrm{H} & 2.81727 & -2.35073 & 0.05846 \\ \mathrm{H} & 4.36654 & 1.38223 & -0.05264 \\ \mathrm{H} & 2.88453 & 2.37412 & -0.30042 \\ \mathrm{H} & 3.20538 & 1.63643 & 1.30140 \\ \mathrm{H} & 3.88695 & -0.06543 & -2.23155 \\ \mathrm{H} & 2.37318 & -1.01147 & -2.53445 \\ \mathrm{H} & 2.34183 & 0.77409 & -2.62977 \\ \mathrm{H} & -4.33252 & 1.52357 & 0.16308 \\ \mathrm{H} & -3.20580 & 1.55256 & 1.56204 \\ \mathrm{H} & -2.81324 & 2.48548 & 0.08815 \\ \mathrm{H} & -4.39488 & -1.24483 & 0.02567 \\ \mathrm{H} & -2.90294 & -2.23954 & -0.20429 \\ \mathrm{H} & -3.22926 & -1.48714 & 1.38273 \\ \mathrm{H} & -3.90481 & 0.31807 & -2.15172 \\ \mathrm{H} & -2.34966 & 1.18081 & -2.44970 \\ \mathrm{H} & -2.40777 & -0.60408 & -2.57880\end{array}$




\section{$\mathrm{S}_{3} \mathrm{Cl}_{3}$}

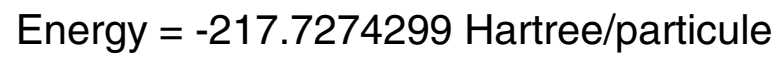

$\begin{array}{rrrr}34 & & & \\ \mathrm{Ru} & 0.00163 & -0.01234 & -0.00058 \\ \mathrm{Si} & -0.00496 & 0.01308 & 2.26963 \\ \mathrm{P} & 2.35851 & -0.00002 & -0.23371 \\ \mathrm{P} & -2.34810 & -0.11766 & -0.27292 \\ \mathrm{Cl} & 0.06603 & -2.26599 & -0.75785 \\ \mathrm{Cl} & -1.67276 & 0.85685 & 3.24942 \\ \mathrm{Cl} & 0.07866 & -1.92433 & 3.04943 \\ \mathrm{Cl} & 1.57287 & 1.00549 & 3.25819 \\ \mathrm{C} & -3.34804 & 1.40072 & 0.04329 \\ \mathrm{C} & -3.25758 & -1.44848 & 0.61626 \\ \mathrm{C} & -2.73605 & -0.46973 & -2.04613 \\ \mathrm{C} & 2.79205 & -0.35080 & -1.99650 \\ \mathrm{C} & 3.31754 & -1.27476 & 0.68481 \\ \mathrm{C} & 3.27734 & 1.56962 & 0.07945 \\ \mathrm{H} & -0.01606 & 1.55545 & -0.39338 \\ \mathrm{H} & -0.06852 & 1.47666 & 0.66553 \\ \mathrm{H} & -3.24236 & 1.70135 & 1.09842 \\ \mathrm{H} & -2.97286 & 2.21865 & -0.59697 \\ \mathrm{H} & -4.41562 & 1.22477 & -0.18261 \\ \mathrm{H} & -3.24457 & -1.24256 & 1.69989 \\ \mathrm{H} & -4.30406 & -1.51479 & 0.26639 \\ \mathrm{H} & -2.73745 & -2.40353 & 0.43119 \\ \mathrm{H} & -3.82885 & -0.50946 & -2.21086 \\ \mathrm{H} & -2.30290 & 0.32021 & -2.68530 \\ \mathrm{H} & -2.28077 & -1.43558 & -2.32226 \\ \mathrm{H} & 2.38792 & -1.34011 & -2.26883 \\ \mathrm{H} & 2.33216 & 0.41094 & -2.65107 \\ \mathrm{H} & 3.88804 & -0.33937 & -2.14291 \\ \mathrm{H} & 3.28698 & -1.05187 & 1.76472 \\ \mathrm{H} & 2.84016 & -2.25414 & 0.51228 \\ \mathrm{H} & 4.36869 & -1.30180 & 0.34394 \\ \mathrm{H} & 3.14260 & 1.87639 & 1.12949 \\ \mathrm{H} & 4.35527 & 1.44456 & -0.13106 \\ \mathrm{H} & 2.87072 & 2.36061 & -0.57527\end{array}$




\section{S2}

Energy $=-169.3740437$ Hartree/particule

31

$\begin{array}{crrr}\mathrm{Ru} & 0.00068 & -0.02265 & 0.01712 \\ \mathrm{H} & -0.00223 & 0.05470 & 1.56990 \\ \mathrm{P} & 2.33406 & -0.00410 & 0.06935 \\ \mathrm{P} & -2.33056 & -0.13449 & 0.05477 \\ \mathrm{Cl} & 0.06617 & -2.35629 & -0.58345 \\ \mathrm{H} & -0.04514 & 1.41167 & 0.70583 \\ \mathrm{H} & -0.03732 & 1.47749 & -0.51355 \\ \mathrm{C} & 3.08074 & -1.24199 & 1.21791 \\ \mathrm{C} & 3.17579 & 1.56630 & 0.56654 \\ \mathrm{C} & 3.16301 & -0.40684 & -1.53448 \\ \mathrm{C} & -3.12431 & -0.57223 & -1.55753 \\ \mathrm{C} & -3.26156 & 1.38364 & 0.55593 \\ \mathrm{C} & -3.01745 & -1.41851 & 1.19003 \\ \mathrm{H} & -4.35492 & 1.21841 & 0.55092 \\ \mathrm{H} & -2.94406 & 1.68275 & 1.57047 \\ \mathrm{H} & -3.01514 & 2.20600 & -0.13878 \\ \mathrm{H} & -4.22235 & -0.66678 & -1.46461 \\ \mathrm{H} & -2.88727 & 0.20486 & -2.30593 \\ \mathrm{H} & -2.69667 & -1.52997 & -1.90154 \\ \mathrm{H} & -4.12000 & -1.47778 & 1.12858 \\ \mathrm{H} & -2.57119 & -2.38959 & 0.91493 \\ \mathrm{H} & -2.71798 & -1.18139 & 2.22591 \\ \mathrm{H} & 4.27636 & 1.46021 & 0.57481 \\ \mathrm{H} & 2.89323 & 2.36771 & -0.13880 \\ \mathrm{H} & 2.83112 & 1.85698 & 1.57460 \\ \mathrm{H} & 4.18516 & -1.24531 & 1.16209 \\ \mathrm{H} & 2.76433 & -1.01256 & 2.25050 \\ \mathrm{H} & 2.68584 & -2.23666 & 0.94840 \\ \mathrm{H} & 4.26424 & -0.43480 & -1.43482 \\ \mathrm{H} & 2.79573 & -1.39098 & -1.87345 \\ \mathrm{H} & 2.88448 & 0.34880 & -2.29055\end{array}$




\section{$\mathrm{TS} \mathrm{Me}_{2} \mathrm{Cl}$}

Energy = -202.6792774 Hartree/particule

Imaginary frequency $=-31.1 \mathrm{~cm}^{-1}$

40

$\begin{array}{cccc}\mathrm{C} & 0.00000 & 0.00000 & 0.00000 \\ \mathrm{Si} & 0.00000 & 0.00000 & 1.89343 \\ \mathrm{C} & 1.80821 & 0.00000 & 2.45382 \\ \mathrm{Cl} & -0.56882 & -2.03613 & 2.29344 \\ \mathrm{Ru} & -1.61846 & 1.46686 & 2.75856 \\ \mathrm{P} & -1.51691 & 3.18049 & 1.13510 \\ \mathrm{C} & -2.29674 & 2.92087 & -0.51810 \\ \mathrm{P} & -2.03249 & 0.21522 & 4.70499 \\ \mathrm{C} & -3.18258 & -1.22294 & 4.62965 \\ \mathrm{Cl} & -3.97596 & 1.36125 & 2.31442 \\ \mathrm{C} & -0.61697 & -0.42660 & 5.70296 \\ \mathrm{C} & -2.89357 & 1.31543 & 5.92015 \\ \mathrm{C} & -2.44968 & 4.64839 & 1.76272 \\ \mathrm{C} & 0.12414 & 3.92507 & 0.71769 \\ \mathrm{H} & -0.90331 & 2.42914 & 3.76283 \\ \mathrm{H} & -0.04219 & 1.62570 & 2.95628 \\ \mathrm{H} & -0.09269 & -1.20988 & 5.12922 \\ \mathrm{H} & -0.96416 & -0.85282 & 6.66218 \\ \mathrm{H} & 0.08698 & 0.39938 & 5.90650 \\ \mathrm{H} & -3.13980 & 0.76971 & 6.85006 \\ \mathrm{H} & -2.25045 & 2.17931 & 6.16303 \\ \mathrm{H} & -3.82293 & 1.68846 & 5.45597 \\ \mathrm{H} & -3.42405 & -1.58416 & 5.64637 \\ \mathrm{H} & -2.71802 & -2.03337 & 4.04540 \\ \mathrm{H} & -4.10362 & -0.89916 & 4.11646 \\ \mathrm{H} & -2.00828 & 4.98391 & 2.71745 \\ \mathrm{H} & -3.49308 & 4.34131 & 1.94865 \\ \mathrm{H} & -2.43018 & 5.48206 & 1.03620 \\ \mathrm{H} & -2.35152 & 3.86692 & -1.08763 \\ \mathrm{H} & -3.31331 & 2.52431 & -0.35416 \\ \mathrm{H} & -1.71925 & 2.18048 & -1.09578 \\ \mathrm{H} & 0.01861 & 4.79316 & 0.04135 \\ \mathrm{H} & 0.76846 & 3.17135 & 0.23333 \\ \mathrm{H} & 0.61523 & 4.25061 & 1.65153 \\ \mathrm{H} & 0.40837 & 0.93979 & -0.41629 \\ \mathrm{H} & -1.02015 & -0.14889 & -0.39709 \\ \mathrm{H} & 0.63147 & -0.83494 & -0.36027 \\ \mathrm{H} & 2.30704 & 0.94767 & 2.16848 \\ \mathrm{H} & 1.89584 & -0.10910 & 3.55047 \\ \mathrm{H} & 2.35912 & -0.83640 & 1.98366\end{array}$




\section{$\mathrm{TS} 3 \mathrm{MeCl}_{2}$}

Energy = -210.204681 Hartree/particule

Imaginary frequency $=-28.1 \mathrm{~cm}^{-1}$

37

$\begin{array}{llll}\text { C } & 0.00000 & 0.00000 & 0.00000\end{array}$

$\begin{array}{llll}\text { Si } & 0.00000 & 0.00000 & 1.88456\end{array}$

$\begin{array}{llll}\mathrm{Cl} & 2.08462 & 0.00000 & 2.35474\end{array}$

$\begin{array}{llll}\text { Cl } & -0.53058 & -2.01700 & 2.29731\end{array}$

$\begin{array}{llll}\mathrm{Ru} & -1.29228 & 1.52379 & 3.01415\end{array}$

$\begin{array}{llll}P & -1.31287 & 3.21712 & 1.37141\end{array}$

$\begin{array}{llll}\text { C } & -2.21266 & 2.98233 & -0.22170\end{array}$

$\begin{array}{llll}\mathrm{P} & -1.52156 & 0.24539 & 4.98840\end{array}$

$\begin{array}{llll}\text { C } & -2.71136 & -1.15752 & 5.04095\end{array}$

$\begin{array}{llll}\mathrm{Cl} & -3.55762 & 1.05737 & 2.46271\end{array}$

$\begin{array}{llll}\text { C } & -0.00511 & -0.44261 & 5.78388\end{array}$

$\begin{array}{llll}\text { C } & -2.19497 & 1.36268 & 6.30457\end{array}$

$\begin{array}{llll}\text { C } & -2.21205 & 4.66595 & 2.09304\end{array}$

$\begin{array}{llll}\text { C } & 0.29276 & 3.96716 & 0.84847\end{array}$

$\begin{array}{llll}\mathrm{H} & -0.19258 & 2.38704 & 3.84115\end{array}$

$\begin{array}{llll}\mathrm{H} & 0.30744 & 1.72843 & 3.22024\end{array}$

$\begin{array}{llll}H & 0.42867 & -1.21849 & 5.12971\end{array}$

$\begin{array}{llll}\mathrm{H} & -0.23594 & -0.88586 & 6.76998\end{array}$

$\begin{array}{llll}\mathrm{H} & 0.73963 & 0.36242 & 5.91027\end{array}$

$\begin{array}{llll}\mathrm{H} & -2.34101 & 0.81930 & 7.25674\end{array}$

$\begin{array}{llll}H & -1.50193 & 2.20598 & 6.47121\end{array}$

$\begin{array}{llll}\mathrm{H} & -3.16602 & 1.76567 & 5.96660\end{array}$

$\begin{array}{llll}\mathrm{H} & -2.81966 & -1.53594 & 6.07383\end{array}$

$\begin{array}{llll}\mathrm{H} & -2.35214 & -1.96347 & 4.38163\end{array}$

$\begin{array}{llll}\mathrm{H} & -3.68435 & -0.80524 & 4.65992\end{array}$

$\begin{array}{llll}\mathrm{H} & -1.71497 & 4.98842 & 3.02491\end{array}$

$\begin{array}{llll}\mathrm{H} & -3.24265 & 4.35171 & 2.33410\end{array}$

$\begin{array}{llll}\mathrm{H} & -2.24316 & 5.51510 & 1.38506\end{array}$

$\begin{array}{llll}\mathrm{H} & -2.33730 & 3.94685 & -0.74720\end{array}$

$\begin{array}{llll}\mathrm{H} & -3.20038 & 2.54594 & 0.00340\end{array}$

$\begin{array}{llll}\mathrm{H} & -1.66038 & 2.28322 & -0.87016\end{array}$

$\begin{array}{llll}\mathrm{H} & 0.13911 & 4.82659 & 0.17050\end{array}$

$\begin{array}{llll}\mathrm{H} & 0.90952 & 3.20813 & 0.33676\end{array}$

$\begin{array}{llll}\mathrm{H} & 0.84221 & 4.30435 & 1.74473\end{array}$

$\begin{array}{llll}\mathrm{H} & 0.44249 & 0.92215 & -0.41832\end{array}$

$\begin{array}{llll}\mathrm{H} & -1.03730 & -0.10664 & -0.36660\end{array}$

$\begin{array}{llll}\mathrm{H} & 0.59392 & -0.85907 & -0.36290\end{array}$ 
$\mathrm{H}_{2}$

Energy $=-1.1744966$ Hartree/particule

2

$\begin{array}{llll}\mathrm{H} & 0.00000 & 0.00000 & -0.00897\end{array}$

$\begin{array}{llll}\mathrm{H} & 0.00000 & 0.00000 & 0.74897\end{array}$

$\mathrm{HSiMe}_{2} \mathrm{Cl}$

Energy $=-34.4717924$ Hartree/particule

11

$\begin{array}{llll}\text { C } & -0.01773 & -0.00064 & -0.01930\end{array}$

$\begin{array}{llll}\mathrm{Si} & 0.03774 & 0.02444 & 1.85310\end{array}$

$\begin{array}{llll}\text { C } & 1.78504 & -0.00078 & 2.52906\end{array}$

$\begin{array}{llll}\mathrm{H} & 0.49318 & -0.89514 & -0.42106\end{array}$

$\begin{array}{llll}\mathrm{H} & 0.48268 & 0.89777 & -0.42982\end{array}$

$\begin{array}{llll}\mathrm{H} & -1.06121 & -0.00786 & -0.38349\end{array}$

$\begin{array}{llll}\mathrm{H} & 2.33916 & 0.89727 & 2.19349\end{array}$

$\begin{array}{llll}\mathrm{H} & 2.33402 & -0.89561 & 2.18184\end{array}$

$\begin{array}{llll}\mathrm{H} & 1.78069 & -0.00729 & 3.63425\end{array}$

$\begin{array}{llll}\mathrm{Cl} & -0.96948 & -1.66660 & 2.56556\end{array}$

$\begin{array}{llll}\mathrm{H} & -0.71134 & 1.20350 & 2.38304\end{array}$

\section{$\mathrm{HSiMeCl}_{2}$}

Energy $=-41.988362$ Hartree/particule

8

$\begin{array}{rrrr}\mathrm{H} & -0.04699 & 0.00000 & -0.00828 \\ \mathrm{Si} & 0.03284 & 0.00000 & 1.47565 \\ \mathrm{C} & 1.77538 & 0.00000 & 2.13193 \\ \mathrm{H} & 1.77802 & 0.00000 & 3.23680 \\ \mathrm{H} & 2.31517 & 0.89836 & 1.77901 \\ \mathrm{H} & 2.31517 & -0.89836 & 1.77901 \\ \mathrm{Cl} & -0.96810 & 1.68550 & 2.14195 \\ \mathrm{Cl} & -0.96810 & -1.68550 & 2.14195\end{array}$

$\mathrm{HSiCl}_{3}$

Energy $=-49.4980326$ Hartree/particule

5

$\begin{array}{rrrr}\mathrm{H} & 0.00000 & 0.00000 & 1.97965 \\ \mathrm{Si} & 0.00000 & 0.00000 & 0.50128 \\ \mathrm{Cl} & 0.00000 & 1.93555 & -0.17642 \\ \mathrm{Cl} & 1.67624 & -0.96778 & -0.17642 \\ \mathrm{Cl} & -1.67624 & -0.96778 & -0.17642\end{array}$


Table A. Selected bond distances and angles for the DFT/B3LYP optimized geometries of $\mathrm{RuCl}\left(\mathrm{SiMe}_{3-\mathrm{n}} \mathrm{Cl}_{\mathrm{n}}\right)\left(\eta^{2}-\mathrm{H}_{2}\right)\left(\mathrm{PMe}_{3}\right)_{2}\left(\mathrm{~S}_{3} \mathbf{M e}_{3-\mathrm{n}} \mathbf{C l}_{\mathbf{n}}, \mathrm{n}=1-3\right) . \mathrm{R}_{1}$ is the silicon substituent which is in the equatorial plane of the complex. Distances are in $\AA$ angle angles in ${ }^{\circ}$.

\begin{tabular}{|c|c|c|c|c|c|c|c|}
\hline & \multicolumn{2}{|c|}{$\mathrm{S3Me}_{2} \mathrm{Cl}$} & \multicolumn{2}{|c|}{$\mathrm{S} 3 \mathrm{MeCl}_{2}$} & \multicolumn{2}{|c|}{$\mathrm{S3Cl}_{3}$} & \multirow{2}{*}{$\begin{array}{l}\mathrm{RuHCl}\left(\mathrm{H}_{2}\right)\left(\mathrm{PMe}_{3}\right)_{2} \\
\quad\left(\mathrm{Si} \equiv \mathrm{H}_{3}\right)\end{array}$} \\
\hline & $\mathrm{R}_{1}=\mathrm{Me}$ & $\mathrm{R}_{1}=\mathrm{Cl}$ & $\mathrm{R}_{1}=\mathrm{Me}$ & $\mathrm{R}_{1}=\mathrm{Cl}$ & Isonh & Isoғ2 & \\
\hline $\mathrm{Ru}-\mathrm{Cl}_{1}$ & 2.434 & 2.433 & 2.412 & 2.420 & 2.398 & 2.410 & 2.427 \\
\hline $\mathrm{Cl}_{1} \cdots \mathrm{R}_{1}$ & 3.838 & 4.182 & 3.678 & 4.210 & 3.832 & 4.154 & - \\
\hline $\mathrm{Ru}-\mathrm{P}_{1}$ & 2.371 & 2.388 & 2.386 & 2.394 & 2.395 & 2.398 & 2.358 \\
\hline $\mathrm{Ru}-\mathrm{P}_{2}$ & 2.380 & 2.381 & 2.385 & 2.386 & 2.394 & 2.396 & 2.358 \\
\hline $\mathrm{Ru}-\mathrm{H}_{1}$ & 1.605 & 1.630 & 1.648 & 1.625 & 1.657 & 1.620 & 1.616 \\
\hline $\mathrm{Ru}-\mathrm{H}_{2}$ & 1.577 & 1.564 & 1.645 & 1.565 & 1.645 & 1.571 & 1.620 \\
\hline $\mathrm{Si}-\mathrm{R}_{1}$ & 1.896 & 2.153 & 1.883 & 2.131 & 2.096 & 2.107 & - \\
\hline $\mathrm{Si}-\mathrm{R}_{2}$ & 1.891 & 1.890 & 2.138 & 2.126 & 2.119 & 2.105 & - \\
\hline $\mathrm{Si}-\mathrm{R}_{3}$ & 2.152 & 1.890 & 2.138 & 1.881 & 2.119 & 2.105 & - \\
\hline $\mathrm{Si} \cdots \mathrm{Cl}_{1}$ & 3.983 & 4.389 & 3.681 & 4.302 & 3.791 & 4.142 & 3.208 \\
\hline $\mathrm{Ru}-\mathrm{Si}$ & 2.334 & 2.340 & 2.302 & 2.308 & 2.288 & 2.287 & 1.562 \\
\hline $\mathrm{Si}-\mathrm{H}_{1}$ & 1.987 & 1.865 & 2.293 & 1.889 & 2.264 & 1.943 & 1.711 \\
\hline $\mathrm{H}_{1}-\mathrm{H}_{2}$ & 1.453 & 1.671 & 0.997 & 1.587 & 0.989 & 1.441 & 1.093 \\
\hline $\mathrm{H}_{1}-\mathrm{Ru}-\mathrm{H}_{2}$ & 54.3 & 63.0 & 35.3 & 59.6 & 34.5 & 53.7 & 39.5 \\
\hline $\mathrm{H}_{1}-\mathrm{Ru}-\mathrm{Si}$ & 57.0 & 52.4 & 68.7 & 54.1 & 67.9 & 56.6 & 65.1 \\
\hline $\mathrm{R}_{1}-\mathrm{Si}-\mathrm{H}_{1}$ & 148.5 & 137.6 & 156.8 & 142.3 & 154.8 & 148.6 & - \\
\hline $\mathrm{P}_{1}-\mathrm{Ru}-\mathrm{P}_{2}$ & 165.5 & 164.6 & 166.9 & 166.0 & 167.3 & 166.3 & 175.8 \\
\hline $\mathrm{Cl}_{1}-\mathrm{Ru}-\mathrm{H}_{2}$ & 135.5 & 110.9 & 153.4 & 115.3 & 149.3 & 126.0 & 150.4 \\
\hline $\mathrm{Cl}_{1}-\mathrm{Ru}-\mathrm{H}_{1}$ & 170.2 & 173.2 & 171.4 & 175.0 & 175.7 & 179.2 & 170.1 \\
\hline $\mathrm{Cl}_{1}-\mathrm{Ru}-\mathrm{Si}$ & 113.3 & 133.6 & 102.7 & 130.9 & 108.0 & 123.8 & 105.0 \\
\hline $\mathrm{H}_{2}-\mathrm{Ru}-\mathrm{Si}-\mathrm{H}_{1}$ & 4.1 & -0.4 & 1.2 & -2.2 & 2.5 & -1.5 & 0.5 \\
\hline $\mathrm{R}_{1}-\mathrm{Si}-\mathrm{Ru}-\mathrm{H}_{1}$ & 176.5 & 179.9 & 180.0 & 177.4 & 179.8 & 179.2 & - \\
\hline$\underset{\left(\mathrm{kJ} . \mathrm{mol}^{-1}\right)}{\Delta \mathbf{E}_{\text {isom }}}$ & 0.0 & +5.1 & 0.0 & +7.6 & 0.0 & +0.8 & - \\
\hline
\end{tabular}

